\title{
Detection and Classification of Diseases in Cucumber Leaves
}

\author{
RajaKumar.R ${ }^{\mathrm{a}, 1}$, Pandian.R $\mathrm{R}^{\mathrm{b}}$, and PremJacob.T $\mathrm{T}^{\mathrm{c}}$ \\ ${ }^{a} U G$ Scholar, Dept of ECE, Sathyabama Institute of Science and Technology, Chennai, \\ India \\ ${ }^{\mathrm{b}} U G$ Scholar, Dept of ECE, Sathyabama Institute of Science and Technology, Chennai, \\ India \\ ${ }^{\mathrm{c}} U G$ Scholar, Dept of ECE, MIT Campus, Anna University, Chennai, India
}

\begin{abstract}
Leaf diseases in cucumber must be identified early and accurately as it reduces the yield. Disease detection in plants is the important field of Artificial Intelligent in agriculture. This causes a periodic outbreak of plant diseases which leads to crop death. Automatic plant disease detection may be benefits in monitoring large scale cultivation of crops, and detects the disease symptoms at early stage itself. Here to detect the disease present in the cucumber the tensor flow technique is used and then artificial neural network is used to classify these diseases. The common disease like cucumber mosaic virus, leaf miner, bacterial leaf spot, and leaf blight are examined with disease detection accuracy of $98.66 \%$. This image processing techniques and algorithm are designed using python language to segment the diseases and categories them whether infected or healthy with the help of Pycharm software.
\end{abstract}

Keywords: Tensor Flow Technique, Artificial Neural Networks (ANN).

\section{Introduction}

In recent years, image processing finds a path in both analog and digital domains with technological development and their requirement. Analog image processing comes under the category of using hardware. Using these visual techniques while interpretation image analysts are various fundamentals. Digital image processing deals with digital images or digital signals by using processors. Image processing is done by using various processes. Some of the main processes involved are image filtering, image classification, and image detection. The image is processed using various principles and algorithms. In India, the production of cucumber is more in southern states due to moderate climatic conditions. Cucumber contains higher water content which helps to avoid dehydration during summer seasons. It also finds a place in cosmetics and medicines The production and consumption of cucumber differ from country to country. The major advantages of cucumber are, Cost is cheaper, rich in nutritional value, the investment cost is lower and higher profit.

\footnotetext{
${ }^{1}$ RajaKumar.R, UG Scholar, Dept of ECE, Sathyabama Institute of Science and Technology, Chennai, India. E-mail: rrkmird@gmail.com
} 
Disease mitigation is the prime factor to be considered in the case of farming practices. So immediate attention should be given to crops which get affected. On observation by naked eyes, it is difficult to recognize the disease in leaf, this might result in the wrong application of pesticides and ultimately it results in crop failure. Hence an automatic system that can detect diseases is mandatory since this system will be useful in monitoring the crops and hence immediate actions can be taken. It is found that image processing techniques will give successful results in disease detection.

Considering this crop because in India up to $65 \%$ of total cucumber production are produced in Karnataka which also includes Andhra Pradesh and Tamil Nadu. Leaf diseases in cucumber must be identified early as it reduces the yield. If we have a proper monitoring system for farming it is possible to produce healthy cucumber plant Main solution is to create a system which can detect the diseases present in cucumber leaf using tensor flow technique which is the first order of business and the second level is to classify them using artificial neural network.

\section{Related Works}

At present many types of research have been carried out in digital image processing particularly in the field of agriculture. We have discussed some of the existing algorithms in this section. In 2019, AbiramiDevaraj et.al [1], described the method of finding and classifying the diseases in cotton leaves. Nearly $80-90 \%$ of the leaf diseases that arise in cotton plants are.,(i) A fungal disease named Alternaria leaf spot and Cercospora leaf spot (ii) An Bacterial diseases named Bacterial delight and (iii) Red and brown spots. They used many image processing techniques and ideas to find and classify the diseases in cotton leaves.In 2019, Santhosh Kumar S et.al [2] In order to increase agricultural income and production, devised a system for identifying diseases in plants. It needs a remarkable amount of labor suite a lot of experience with plant diseases and extreme time. Image is processed sing various algorithm.In 2018,M.S.Arya et.al [3] proposed a most significant system for predicting diseases in paddy crops. As we know that consumption of this crop and their need is more in our country. So farmers find difficulties in identifying the diseases. They used some image processing ideas to detect the diseases in the paddy field.In 2017, V. Pooja et.al [4] In recent days most of the plants suffer from many diseases due to changing climatic conditions, unpredictable rain, Pollution, and other environmental impacts. They proposed a paper based on a survey of existing plant diseases and their detection process. Three important steps comprising of pre-processing and analysis of the image, Recognition of plant diseases, and feature extraction is employed here.In 2016 Anand et al [5] proposed a diagnostic mechanism that captures a disease-affected leaf accurately using some algorithms and techniques. They also used ANN Classification techniques for disease identification in plants for its accuracy. In this system features such as HSV and color are extracted in the Segmentation process.In 2015 Y.Sanjana et.al [6] they proposed a system in which mobile phones capture the agriculture field and sent that images to the server for processing. In this method, the classification and detection of leaf diseases are done by using Computer Vision Technology(CVT). They provide a simple approach for the identification of diseases in plants.In 2013 Prof Sanjay B. Dhaygude et.al [7] Mostly diseases fungi, bacteria, and viruses are responsible for diseases in plants. The fungal diseases are caused by reproductive structures in plants and also due to their morphology 
[14-15]. These diseases spread to other plants or leaves by means of water, air, movement of contaminated soil or animals. So they proposed a Color transformation structure to detect leaf diseases. Here they used HSV instead of RGB due to its good color descriptor property. In this paper, Section I gives the Introduction part, Section II deals with existing works and their methods, Section III explains the diseases in cucumber leaf, Section IV provides the methodology and the techniques used, Section V gives the output results and their future scope.

\section{Proposed Work}

Step 1: The diseased leaf images are captured using high resolution camera, which the back-ground must be constant black, so it gets isolated from complex back-ground. The main advantage of digital image is that it makes more number of copies without any loss of image quality. Step 2: Then comes to pre-processing, where the conversion of RGB color to grey scale images are produced. The only reason for these conversion is that the RGB image require more space and also consumes larger time period. Step 3: Image segmentation is the method of partitioning a digital image into multiple number of sets of pixels, which should be only done with morphological operation. Step 4: Feature extraction is that the extracted feature is fed into the feature training process, that is done with the help of tensor flow technique. The feature having negative value (-1) as output is represented as diseased feature whereas positive value $(+1)$ is belong to the healthy or normal feature set.

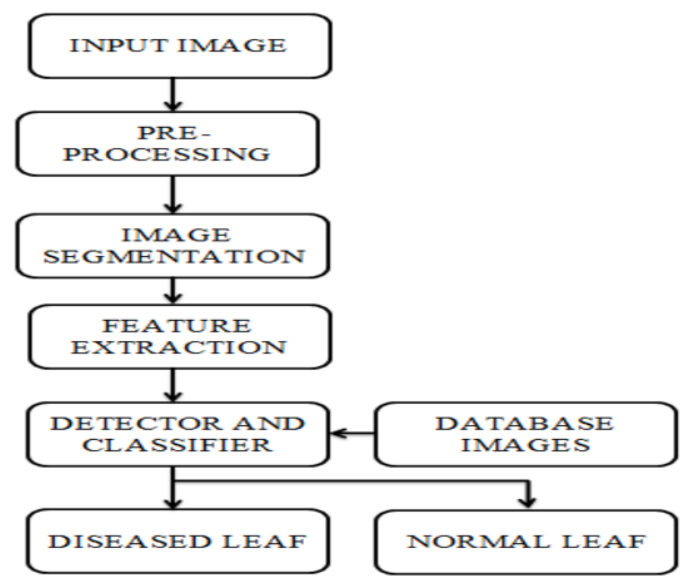

Figure1.Block Diagram

\section{Methodology}

Tensor flow can train and run deep neural network for image recognition-based simulations. It supports production prediction with the same model that is used for the training. Some of the imports used in the model are given below:

1. cv2 2. NumPy 3. OS \& tflearn. 


\subsection{Classification using ANN:}

There are four classes for cucumber crop disease classification they are bacterial leaf spot, cucumber mosaic virus, leaf miner and healthy leaf.

\subsection{Python programming:}

The very first step is to import the function for pooling and then importing the function for convolution. And to load and reshape the data, here we have imported a fully-connected function and regression function. Here to convert the images and then labels to array information is the primary step after the constants are imported.With the help of the tqdm model, we can know about our process by giving the progress bar. Training and testing data are the labeled data sets, where the neural network will fit in the training data, and to test the accuracy of the network the testing data will be used.

\section{Results and Future Scope}

Training a neural network takes more than a few epochs. The main goal of the model is to classify and detect diseased leaves. With the help of global features extracted from the images like shape, color, and texture feature, the model will be able to differentiate the diseased leave from that of the normal leaf. Relu(Rectified Linear Unit) is a good hidden layer activation choice because it overcomes the weakness and does not suffer from it like sigmoid and tanh. It will control the input value and helps to forward to the next level.

Table 1.parameters used in the model

\begin{tabular}{|c|l|}
\hline Parameter & \multicolumn{1}{|c|}{ Value } \\
\hline Epochs & 8 \\
\hline Learning rate & le-3 \\
\hline Activationin middle layers & Relu \\
\hline Activation in Final layer & Softmax \\
\hline
\end{tabular}

Softmax is a normalized exponential function that will output from a given set of $\mathrm{N}$ inputs a probability distribution to each input. About $80 \%$ of the given dataset used for training the dataset and the remaining $20 \%$ of the dataset used for testing. Here the testing dataset will be used to test the accuracy of the network.
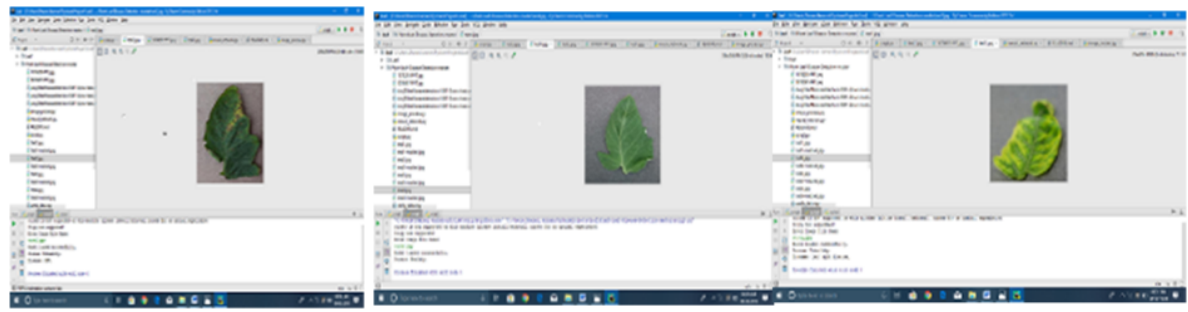

Figure 2.a)leaf infected b)output for leaf with CMV 3c)Healthy leaf 
In this paper, we analyzed the diseases that present in cucumber leaves. By using Artificial Neural Network(ANN) techniques we classified the diseases that are present in cucumber leaf. First, the preprocessing of the leaf is performed, and then disease affected leaf is considered for the tensor flow diagram. The common diseases in cucumber such as leaf miner, leaf spot, and CMV are considered for experimental analysis. The future scope of our work is, with the help of drones or cameras, the large agricultural land is monitored at constant intervals of time and instant information about the farmland is sent to the farmers via mobile apps or SMS. From that farmers can predict the diseases at an early time which helps to avoid larger loss.

\section{References}

[1] AbiramiDevaraj, KarunyaRathan,SarvepalliJaahnavi, K Indira. Identification of Plant Disease using Image Processing Technique .2019, International Conference on Communication and Signal Processing (ICCSP).

[2] Santhosh Kumar S, B. K. Raghavendra .Diseases Detection of Various Plant Leaf Using Image Processing Techniques: A Review.2019, 5th International Conference on Advanced Computing \& Communication Systems (ICACCS).

[3] Arya, K Anjali, DivyaUnni .Detection of unhealthy plant leaves using image processing and genetic algorithm with Arduino. 2018, International Conference on Power, Signals, Control and Computation (EPSCICON).

[4] Pooja, Rahul Das, V Kanchana. Identification of plant leaf diseases using image processing techniques .2017, IEEE Technological Innovations in ICT for Agriculture and Rural Development (TIAR).

[5] Anand, S Veni, J Aravinth. An application of image processing techniques for detection of diseases on brinjal leaves using k-means clustering method. 2016, International Conference on Recent Trends in Information Technology (ICRTIT).

[6] Sanjana, AshwathSivasamy, SriJayanth. Plant disease detection using image processing techniques. International Journal of Innovative Research in Science, Engineering and Technology, Vol. 4, Special Issue 6, May 2015.

[7] Sanjay B. Dhaygude, Mr.NitinP.Kumbhar. Agricultural plant leaf disease detection using image processing. International Journal of Advanced Research in Electrical, Electronics and Instrumentation Engineering Vol. 2, Issue 1, January 2013.

[8] Pramod S. landge, Sushil A. Patil, Dhanashree S. Khot .Automatic detection and classification of plant disease through image processing .International Journal of Advanced Research in Computer Science and Software Engineering, Volume 3, Issue 7, 2013.

[9] Heeb Al Bashish, Malik Braik, and SuliemanBani-Ahmad .A Framework for detection and classification of plant leaf and stem diseases. IEEE 2010.

[10] ZhangJian and Zhang Wei .Support vector machine for recognition of cucumber leaf diseases, in Advanced Computer Control (ICACC). 20102nd International Conference on, 2010, vol. 5. IEEE.

[11] SatishMadhgoria, MarekSchikora, and Wolfgang Koch .Pixel-based classification method for detecting unhealthy regions in leaf images. 2009.

[12] Soman, R. Loganathan, and V. Ajay. Machine learning with SVM and other kernel methods, in PHI Learning Pvt. Ltd., 2009.

[13] SantanuPhadikar and Jaya Sil, Rice disease identification using pattern recognition techniques, in Computer and Information Technology, 2008. ICCIT 2008.11th International Conference on.IEEE, 2008 .

[14] Ambeth Kumar.V.D, S. Sharmila, Abhishek Kumar, A. K. Bashir, Mamoon Rashid, Sachin Kumar Gupta \&Waleed S. Alnumay .A novel solution for finding postpartum haemorrhage using fuzzy neural techniques .Neural Computing and Applications (2021) (https://doi.org/10.1007/s00521-020$05683-z)$

[15] Ambeth Kumar.V.D, Malathi.S, Ashok Kumar.V.D (2015) .Performance Improvement Using an Automation System for Segmentation of Multiple Parametric Features Based on Human Footprint. for the Journal of Electrical Engineering \& Technology (JEET), vol. 10, no. 4, pp.1815-1821 , 2015. [http://dx.doi.org/10.5370/JEET.2015.10.4.1815] 